\title{
Autoregression-Based Estimation of the New Keynesian Phillips Curve
}

\author{
Markku Lanne \\ University of Helsinki and HECER \\ and \\ Jani Luoto \\ University of Helsinki and HECER
}

Discussion Paper No. 321

March 2011

ISSN 1795-0562

HECER - Helsinki Center of Economic Research, P.O. Box 17 (Arkadiankatu 7), FI-00014 University of Helsinki, FINLAND, Tel +358-9-191-28780, Fax +358-9-191-28781, E-mail info-hecer@helsinki.fi, Internet www.hecer.fi 


\title{
Autoregression-Based Estimation of the New Keynesian Phillips Curve*
}

\begin{abstract}
We propose an estimation method of the new Keynesian Phillips curve (NKPC) based on a univariate noncausal autoregressive model for the inflation rate. By construction, our approach avoids a number of problems related to the GMM estimation of the NKPC. We estimate the hybrid NKPC with quarterly U.S. data (1955:1--2010:3), and both expected future inflation and lagged inflation are found important in determining the inflation rate, with the former clearly dominating. Moreover, inflation persistence turns out to be intrinsic rather than inherited from a persistent driving process.
\end{abstract}

JEL Classification: C22, C51, E31

Keywords: noncausal time series, non-Gaussian time series, inflation, Phillips curve.

\author{
Markku Lanne \\ Department of Political and Economic \\ Studies \\ University of Helsinki \\ P.O. Box 17 (Arkadiankatu 7) \\ FI-00014 University of Helsinki \\ FINLAND
}

e-mail: markku.lanne@helsinki.fi
Jani Luoto

Department of Political and Economic

Studies

University of Helsinki

P.O. Box 17 (Arkadiankatu 7),

FI-00014 University of Helsinki

FINLAND

e-mail: jani.luoto@helsinki.fi

\footnotetext{
* We are would like to thank Antti Ripatti, Pentti Saikkonen, and Arto Luoma for useful comments. The usual disclaimer applies. Financial support from the Academy of Finland, the OP-Pohjola Group Research Foundation and the Yrjö Jahnsson Foundation is gratefully acknowledged. Part of this research was done while Luoto was visiting the Faculty of Economics of the University of Cambridge.
} 


\section{Introduction}

According to the new Keynesian Phillips curve (NKPC), the inflation rate $\pi_{t}$ depends linearly on the expected inflation rate next period, $E_{t} \pi_{t+1}$, and a measure of marginal costs, $x_{t}$. This equation is a central building block of modern macroeconomic models, and it can be derived from several sets of microfoundations, although probably most often it is attributed to Calvo's (1983) price-setting model where only a fraction of firms can change prices in a given period (or equivalently, each firm is able to adjust its price with a fixed probability). Incorporating lagged inflation $\pi_{t-1}$ into this equation has typically been found to improve the empirical fit, and Galí and Gertler (1999) called this augmented equation the hybrid NKPC. They showed that this version can be obtained by modifying the assumptions of Calvo's (1983) model such that only some firms that are able to change prices, choose to do so optimally, while the rest use a simple rule of thumb based on recent history of aggregate price behavior.

There is an ongoing debate about the importance of forward-looking behavior in the determination of inflation. The issue is particularly important from the viewpoint of monetary policy whose design depends on the sources of inflation persistence. In empirical studies employing univariate methods (see, e.g., Cecchetti and Debelle (2006)), inflation has invariably been found highly persistent, and this persistence has typically been interpreted as dependence on past inflation in forming expectations and, hence, as evidence against the NKPC. Also, Rudd and Whelan (2005a, 2007), and Nason and Smith (2008a), inter alia, have found little evidence of forward-looking inflation dynamics in analyses based on estimated NKPCs for the U.S. On the other hand, the recent results of Lanne and Saikkonen (2011a) and Lanne et al. (2011) based on so-called noncausal autoregressive (AR) models suggest that the persistence in the U.S. inflation results from agents' forward-looking behavior rather than dependence on past inflation. The NKPC estimation results of Galí and Gertler (1999), and Galí 
et al. (2005), to name but a few, also lend support to the NKPC in the U.S.

The principal econometric method used in single-equation estimation of the NKPC is the generalized method of moments (GMM), where various lags of inflation and the marginal cost variable have typically been used as instruments. As already pointed out above, the results have been contradictory. In particular, they seem to strongly depend on the set of instruments and the variable used as a proxy for marginal costs that are not directly observable. Because $\pi_{t}, \pi_{t-1}$, and $x_{t}$ included in the NKPC equation cannot act as instruments for $\pi_{t+1}$, higher-order dynamics are called for, i.e., inflation should be predictable by higher lags of these variables. Alternatively some other variables could be used as instruments, but it is not easy to find variables with predictive power for inflation (see, e.g., Stock and Watson (1999, 2009)). Nason and Smith (2008a) show that lack of higher-order dynamics gives rise to the problem of weak instruments in estimating the NKPC, resulting in weak identification and strong dependence of the results on the choice of instruments. To avoid these problems, they employ methods robust with respect to weak instruments and find little evidence in favor of the hybrid NKPC in U.S. data.

In addition to the problem of weak instruments, there may be another problem hampering the GMM estimation of the NKPC. Namely, Lanne and Saikkonen (2011b) have recently shown that if any of the time series used as instruments is noncausal, i.e., depends on its future values, the GMM estimator is inconsistent. Moreover, in this case, endogeneity of such an instrument is not reliably revealed by Hansen's (1982) $J$ test. Noncausality of inflation found by Lanne and Saikkonen (2011a) and Lanne et al. (2011) thus indicates that using lags of inflation as instruments as is commonly done in the previous literature, is likely to yield misleading results. Lanne and Saikkonen (2011b) also found noncausality very common in a comprehensive data set compirising more than 300 macroeconomic and financial time series, which suggests that finding valid additional instruments for the estimation of the NKPC 
may be challenging.

In this paper, we introduce a single-equation estimator of the parameters of the NKPC based on a noncausal AR model specified for inflation. As discussed in Section 2 below, identification of noncausality requires non-Gaussian errors, and it is this feature that facilitates identification. This is different from the GMM where identification is based on a suitable proxy for the marginal cost variable. Hence, our identification is statistical, with the drawback that is does not directly yield an estimate of the coefficient of the marginal cost. On the other hand, we obtain consistent estimates of the coefficients of lagged and expected future inflation that are independent of any selected marginal cost proxy. Furthemore, leaving a marginal cost proxy unspecified, facilitates reverse-engineering of the process driving inflation consistent with the model.

In short, the benefits of the proposed estimation procedure are twofold. First, no instrumental variables are needed, which abolishes the problems of weak and noncausal instruments prevalent in much of the previous literature. Second, we avoid the difficult problem of finding a proxy for the marginal cost as none is needed. As pointed out by Schorfheide (2008), measurement errors pertaining to the marginal cost series can potentially distort the inference about the NKPC parameters in dynamic stochastic general equilibrium (DSGE) models. We expect this problem to be even more severe in the single-equation setup. Indeed, Nason and Smith (2008b) recently compared the estimates of the U.S. NKPC with nine different marginal cost variables and found that most of them were highly insignificant and greatly affected the values of the parameters of interest. Similarly, Rudd and Whelan (2005b) found that neither labor's share of income nor detrended real GDP provide good proxies for the U.S. marginal cost.

With quarterly U.S. data from 1955:1-2010:3, we demonstrate the problems of the GMM mentioned above. For two inflation measures, we find the best-fitting noncausal 
non-Gaussian AR model. There is strong evidence of deviations from normality of the errors of the estimated AR models. In both cases, the selected model turns out to be mixed, including both lags and leads of inflation. This suggests that both expected future inflation and lagged inflation are important in determining the inflation rate. Estimates of the parameters of the hybrid NKPC based on the noncausal AR models indicate that expected inflation is the dominant factor determining inflation, but backward-looking behavior is not insignificant either. Moreover, inflation persistence is found to follow mostly from agents' forward-looking behavior, while the persistence inherited from the driving variable plays a minor role.

The rest of the paper is structured as follows. Section 2 describes the noncausal AR model of Lanne and Saikkonen (2011a) and discusses model selection. In Section 3, we derive the maximum likelihood estimator of the NKPC based on the selected noncausal AR model for inflation. In Section 4, the empirical results are presented. Finally, Section 5 concludes.

\section{Noncausal Autoregression}

\subsection{Model}

The starting point of our procedure for estimating the NKPC is an adequate noncausal AR model for inflation, and in this section, we briefly describe the noncausal AR model of Lanne and Saikkonen (2011a). Consider a stochastic process $y_{t}(t=0, \pm 1, \pm 2, \ldots)$ generated by

$$
\phi(B) \varphi\left(B^{-1}\right) y_{t}=\epsilon_{t}
$$

where $\phi(B)=1-\phi_{1} B-\cdots-\phi_{r} B^{r}, \varphi\left(B^{-1}\right)=1-\varphi_{1} B^{-1}-\cdots-\varphi_{s} B^{-s}$, and $\epsilon_{t}$ is a sequence of independent, identically distributed (continuous) random variables with mean zero and variance $\sigma^{2}$ or, briefly, $\epsilon_{t} \sim$ i.i.d. $\left(0, \sigma^{2}\right)$. Moreover, $B$ is the usual backward shift operator, that is, $B^{k} y_{t}=y_{t-k}(k=0, \pm 1, \ldots)$, and the polynomials 
$\varphi(z)$ and $\phi(z)$ have their zeros outside the unit circle so that

$$
\phi(z) \neq 0 \text { for }|z| \leq 1 \text { and } \varphi(z) \neq 0 \text { for }|z| \leq 1
$$

We use the abbreviation $\operatorname{AR}(r, s)$ for the model defined by (1). If $\varphi_{1}=\cdots=\varphi_{s}=$ 0 , model (1) reduces to the conventional causal $\mathrm{AR}(r, 0)$ model with $y_{t}$ depending on its past but not future values. We sometimes call this the $\operatorname{AR}(r)$ model. The more interesting cases arise, when this restriction does not hold. If $\phi_{1}=\cdots=\phi_{r}=0$, we have the purely noncausal $\operatorname{AR}(0, s)$ model with dependence on future values only. In the mixed $\operatorname{AR}(r, s)$ case where neither restriction holds, $y_{t}$ depends on its past as well as future values.

A well-known feature of noncausal autoregressions is that a non-Gaussian error term is required to achieve identification. Thus, we assume that the error term $\epsilon_{t}$ is non-Gaussian and that its distribution has a (Lebesgue) density $f_{\sigma}(x ; \boldsymbol{\omega})=$ $\sigma^{-1} f\left(\sigma^{-1} x ; \boldsymbol{\omega}\right)$ which depends on the parameter vector $\boldsymbol{\omega}(d \times 1)$ in addition to the scale parameter $\sigma$ already introduced. The function $f(x ; \boldsymbol{\omega})$ is assumed to satisfy the regularity conditions stated in Andrews et al. (2006) and Lanne and Saikkonen (2011a). These conditions imply that $f(x ; \boldsymbol{\omega})$ is twice continuously differentiable with respect to $(x, \boldsymbol{\omega})$, non-Gaussian, and positive for all $x \in \mathbb{R}$ and all permissible values of $\boldsymbol{\omega}$. For the U.S. inflation we use Student's $t$ distribution as the error distribution in Section 4.

Lanne and Saikkonen (2011a) showed how model (1) can be consistently estimated by the method of maximum likelihood (ML). They also showed that the (local) ML estimator is asymptotically normally distributed, and a consistent estimator of the limiting covariance matrix is obtained in the usual way from the standardized Hessian of the log-likelihood function. Thus, standard errors of estimators and conventional Wald tests with an asymptotic $\chi^{2}$-distribution under the null hypothesis can be constructed as usual. 


\subsection{Model Selection}

In practice the model orders $r$ and $s$ are always unknown and have to be specified based on the data. Because noncausal AR processes are not identified by Gaussian likelihood, the first step in modeling a potentially noncausal time series is to search for signs of nonnormality. To this end, Lanne and Saikkonen (2011a) suggest estimating a Gaussian $\operatorname{AR}(p)$ model that adequately captures the autocorrelation in the series and checking its residuals for nonnormality. As mentioned above, Student's $t$ distribution might be a suitable error distribution for the U.S. inflation as the residuals of Gaussian AR models turn out to be leptokurtic.

Provided nonnormality is detected, the next step is to select the best-fitting model among the alternative $\operatorname{AR}(r, s)$ specifications. As the $\operatorname{AR}(p)$ model has been found to adequately capture the autocorrelation in the series, it seems reasonable to restrict oneself to models with $r+s=p$. Following Breidt et al. (1991), Lanne and Saikkonen (2011a) suggest selecting among these the model that produces the greatest value of the likelihood function. Finally, the adequacy of the selected specification is checked diagnostically and the model is augmented if needed. In addition to examining the fit of the $t$ distribution, Lanne and Saikkonen (2011a) checked the residuals for remaining autocorrelation and conditional heteroskedasticity. The former can conveniently be checked by testing the significance of an additional lead and lag.

\section{Estimation of the New Keynesian Phillips Curve}

In this section, we discuss the different versions of the NKPC and their estimation based on an adequate $\operatorname{AR}(r, s)$ model specified for inflation. The NKPC,

$$
\pi_{t}=\gamma_{f} E_{t} \pi_{t+1}+\lambda x_{t},
$$


incorporates staggered price setting, and it can be derived from a number of different sets of microfoundations, including Calvo's (1983) price-setting model where a fraction of the firms cannot change their prices in a given period. Here $\pi_{t}$ denotes the inflation rate, and $x_{t}$ is a measure of marginal costs. Galí and Gertler (1999) modified Calvo's (1983) model by assuming that some firms able to change prices, choose not to do so. This assumption leads to the so-called hybrid NKPC,

$$
\pi_{t}=\gamma_{f} E_{t} \pi_{t+1}+\gamma_{b} \pi_{t-1}+\lambda x_{t}
$$

that allows for dependence on past inflation. Augmenting the NKPC with $\pi_{t-1}$ has typically been found to improve the empirical fit considerably.

As already pointed out in the Introduction, a major problem and cause of controversy in the empirical implementation of the NKPC is the fact that the marginal cost variable $x_{t}$ is not directly observable. In empirical studies employing the singleequation framework, the most common $x$-variable is the real unit labor cost. A theoretically consistent alternative is the output gap that can be measured in several alternative ways. Arguments in favor of and against both of these variables have been brought up in the previous literature (see, e.g., Nason and Smith (2008) and the references therein). Unfortunately, the choice of the marginal cost proxy greatly affects the estimates and, hence, the assessment of the relative importance of forwardlooking and backward-looking behavior in determining inflation. In our approach, no $x$-variable need be prespecified. This is also possible in the DSGE framework, where $x_{t}$ can be treated as a latent variable, but to our knowledge, this is the first paper to present estimates of the NKPC in the univariate single-equation framework.

Let us first consider the estimation of the hybrid NKPC (4). By adding and subtracting $\gamma_{f} \pi_{t+1}$, equation (4) can be rewritten as

$$
\pi_{t}=\gamma_{f} \pi_{t+1}+\gamma_{b} \pi_{t-1}+\eta_{t+1}
$$


where $\eta_{t+1}=\gamma_{f} E_{t} \pi_{t+1}-\gamma_{f} \pi_{t+1}+\lambda x_{t} \equiv \xi_{t+1}+\lambda x_{t}$, and, as typically done in the rational expectations literature, the expectation error $\xi_{t+1}$ is assumed to be independently and identically distributed (i.i.d.) in time. The time-series properties of $\eta_{t}$ depend on those of $x_{t}$, but we assume that its process can be adequately approximated by a finite-order autoregression. By dividing through by $\gamma_{f}$ and lagging by one period, the model can be written as

$$
\gamma_{f}^{-1} \pi_{t-1}=\pi_{t}+\gamma_{f}^{-1} \gamma_{b} \pi_{t-2}+\gamma_{f}^{-1} \eta_{t}
$$

or, using the backshift operator $B$, as

$$
\left(1-\gamma_{f}^{-1} B+\gamma_{f}^{-1} \gamma_{b} B^{2}\right) \pi_{t}=-\gamma_{f}^{-1} \eta_{t}
$$

The polynomial $a(z) \equiv 1-\gamma_{f}^{-1} z+\gamma_{f}^{-1} \gamma_{b} z^{2}$ can equivalently be written as $a(z)=$ $(1-\phi z)\left(1-\varphi^{*} z\right)$, where

$$
\phi=\frac{1}{2}\left(\gamma_{f}^{-1}-\sqrt{\gamma_{f}^{-2}-4 \gamma_{f}^{-1} \gamma_{b}}\right) \quad \text { and } \quad \varphi^{*}=\frac{1}{2}\left(\gamma_{f}^{-1}+\sqrt{\gamma_{f}^{-2}-4 \gamma_{f}^{-1} \gamma_{b}}\right)
$$

are the characteristic roots of equation (5). With plausible values of $\gamma_{f}$ and $\gamma_{b}, \phi$ is smaller and $\varphi^{*}$ is greater than unity in absolute value (cf. Galí and Gertler (1999) and Galí et al. (2005)). It is now convenient to write the polynomial $a(z)$ as

$$
(1-\phi z)\left(1-\varphi^{*} z\right)=-(1-\phi z) \varphi^{*} z\left(1-\frac{1}{\varphi^{*}} z^{-1}\right)=-\varphi^{*}(1-\phi z) z\left(1-\varphi z^{-1}\right),
$$

where $\varphi^{*}=1 / \varphi$. Subsituting this into (5) yields

$$
(1-\phi B)\left(1-\varphi B^{-1}\right) \pi_{t}=\epsilon_{t}
$$

where $\epsilon_{t} \equiv\left(\varphi^{*} \gamma_{f}\right)^{-1} \eta_{t+1}$. If $\eta_{t}$ were i.i.d., this would be the $\operatorname{AR}(1,1)$ model of Lanne and Saikkonen (2011a) described in Section 2, and consistent estimates of the parameters of the NKPC would be obtained by estimating an $\mathrm{AR}(1,1)$ model for inflation by the method of maximum likelihood (ML) and solving $\gamma_{f}$ and $\gamma_{b}$ from equations 
(6). As long as the expression under the square root in (6) is positive, $\phi$ and $\varphi^{*}$ are real and distinct, and $\gamma_{f}$ and $\gamma_{b}$ are uniquely identified. The shaded area in Figure 1 contains the admissible values of $\gamma_{f}$ and $\gamma_{b}$, and it is seen to cover virtually all reasonable combinations of the values of these parameters. The figure also incorporates the restrictions implied by the structural models undelying the hybrid NKPC, including the model of Galí and Gertler (1999), that $\gamma_{f}$ and $\gamma_{b}$ should lie between zero and one. The consistency of this estimator is guaranteed by the consistency of the ML estimator of the $\operatorname{AR}(1,1)$ model under the general conditions in Lanne and Saikkonen (2011a) that, in particular, assume the adequacy of the AR specification.

Because the marginal cost variable $x_{t}$ is not likely to be i.i.d., the approach above must be modified to allow $\eta_{t}$ to be autocorrelated. This is suggested by the persistence of the theoretically implied variables driving inflation. As already pointed out, we assume the autocorrelation in the error term to be adequately captured by a (potentially noncausal) $\operatorname{AR}(r-1, s-1)$ process, i.e.,

$$
\rho(B) \theta\left(B^{-1}\right) \eta_{t}=\zeta_{t}
$$

where $\rho(B)=1-\rho_{1} B-\cdots-\rho_{r-1} B^{r-1}, \theta\left(B^{-1}\right)=1-\theta_{1} B^{-1}-\cdots-\theta_{s-1} B^{-s+1}$ and $\zeta_{t}$ is an i.i.d. error term. Substituting this into (7) yields

$$
\rho(B) \theta\left(B^{-1}\right)(1-\phi B)\left(1-\varphi B^{-1}\right) \pi_{t}=\varepsilon_{t}
$$

or

$$
\phi(B) \varphi\left(B^{-1}\right) \pi_{t}=\varepsilon_{t},
$$

where $\phi(B) \equiv \rho(B)(1-\phi B), \varphi\left(B^{-1}\right) \equiv \theta\left(B^{-1}\right)\left(1-\varphi B^{-1}\right)$, and $\varepsilon_{t} \equiv\left(\varphi^{*} \gamma_{f}\right)^{-1} \zeta_{t+1}$. This is the $\operatorname{AR}(r, s)$ model of Lanne and Saikkonen (2011a) (cf. model (1)), and ML estimation under the constraints (6) yields consistent estimates of $\gamma_{f}$ and $\gamma_{b}$. Equation (8) may have multiple real characteristic roots, i.e., the parameters $\phi$ and $\varphi^{*}$ are not necessarily unique, but any real characteristic roots may be paired to solve for 
$\gamma_{f}$ and $\gamma_{b}$ in (6). In this case, identification may for practical purposes be attained by restrictions arising from economic theory. For instance, negative values of $\gamma_{f}$ and $\gamma_{b}$ as well as values exceeding unity are precluded. The admissible combinations of these parameters are thus found in the shaded region of Figure 1.

Also the estimation of the purely forward-looking NKPC (3), can be based on a univariate noncausal AR model for inflation. In this case where $\gamma_{b}=0$, equation (5) simplifies to

$$
\left(1-\gamma_{f}^{-1} B\right) \pi_{t}=-\gamma_{f}^{-1} \eta_{t}
$$

and the polynomial $a(z)=1-\gamma_{f}^{-1} z=1-\varphi^{*} z=-\varphi^{*} z\left(1-\varphi z^{-1}\right)$, where $\varphi^{*}=\gamma_{f}^{-1}$. Substituting this into (9) yields

$$
\left(1-\varphi B^{-1}\right) \pi_{t}=\epsilon_{t}
$$

where $\epsilon_{t}=\left(\varphi^{*} \gamma_{f}\right)^{-1} \eta_{t+1}=\eta_{t+1}$ and $\gamma_{f}=\varphi$. Assuming, as above, that $\eta_{t}$ follows a (potentially noncausal) AR process, equation (10) becomes the $\operatorname{AR}(r, s)$ model of Lanne and Saikkonen (2011a),

$$
\phi(B) \varphi\left(B^{-1}\right) \pi_{t}=\varepsilon_{t},
$$

where $\varepsilon_{t}$ is an i.i.d. error term, $\phi(B)=1-\phi_{1} B-\cdots-\phi_{r} B^{r}$ and $\varphi\left(B^{-1}\right)=$ $1-\varphi_{1} B^{-1}-\cdots-\varphi_{s} B^{-s}$. A consistent estimate of $\gamma_{f}$ is obtained as one of the estimated real roots of the polynomial $\varphi\left(z^{-1}\right)$. Like in the case of the hybrid NKPC, $\gamma_{f}$ is not, in general, uniquely identified without further restrictions, and restrictions from economic theory may help eliminate some candidate values.

Notice that the orders of the selected $\operatorname{AR}(r, s)$ model for inflation may, as such, preclude the forward-looking or hybrid NKPC. If $r$ turns out to be zero, the hybrid NKPC is not a possibility, and inflation is purely forward-looking. Conversely, if the best-fitting model is an $\operatorname{AR}(r, 0)$ model, inflation necessarily only depends on 
the past. Hence, successful model selection is of crucial importance for conclusions concerning the nature of inflation dynamics.

\section{Empirical Results}

We provide estimates of the U.S. NKPC based on the GMM and the methods introduced in Section 3. Our quarterly data set covers the period from 1955:1 to 2010:3. Inflation is computed as $\pi_{t}=400 \ln \left(P_{t} / P_{t-1}\right)$, where $P_{t}$ is either the implicit price deflator of the GDP or the consumer price index for all consumers. The

resulting inflation series are denoted by $\pi_{t}^{G D P}$ and $\pi_{t}^{C P I}$, respectively. Following the previous literature, as proxies for the marginal cost we use the real unit labor cost and linearly detrened logarithmic real GDP per capita. The former is computed as $100(1+q) \ln \left(C O M P F N F B_{t} / O P H N F B_{t}\right)-100 \ln P_{t}$, where COMPFNFB $B_{t}$ is the index of hourly compensation in the non-farm business sector, $O P H N F B_{t}$ is the output per hour of all persons in the non-farm business sector, and $q$ is a function of the steady-state markup and labor's share parameter in the firm's production function. Following Nason and Smith (2008a), we set $1+q=1.08$. Despite the fact that both of these variables have been criticized as drivers of inflation (see, e.g., Galí and Gertler (1999) and Rudd and Whelan (2005b)), they are still commonly used in the empirical literature. As additional instruments in GMM estimation, we use lags of wage inflation $\left(w i_{t}\right)$, commodity price inflation computed from the producer price index $\left(c p_{t}\right)$ and the spread between the five-year Treasury constant-maturity interest rate and the 90-day Treasury bill rate $\left(t s_{t}\right)$. The source of all data is the Federal Reserve Bank of St. Louis FRED databank. 


\subsection{GMM Estimation}

To illustrate the pontential problems with GMM estimation of the NKPC alluded to in the Introduction, we first consider GMM estimates for the different inflation and marginal cost series based on alternative sets of instruments. The results are shown in Table 1, and they reconfirm a number of conclusions already drawn in the previous literature (cf., e.g., Nason and Smith (2008b) who present similar results for $\pi_{t}^{G D P}$ using a larger collection of instrument sets). First, the estimated coefficients, their statistical significance and even their signs vary from one instrument set to another. Second, the results vary depending on the marginal cost proxy being used. With the unit labor cost, $\gamma_{f}$ is always significant at conventional significance levels, but with the detrended output only for some instrument sets. Third, different inflation measures seem to produce somewhat different results. In conclusion, it appears to be difficult to obtain general results concerning the issue of forward-looking vs. backward-looking inflation dynamics using the GMM. The $J$ test of overidentifying restrictions (not reported) does not reject at conventional significance levels in any of the cases, but noncausality and, thus, endogeneity of the instruments cannot be precluded. Therefore, we next turn to the estimates of the NKPC based on potentially noncausal inflation dynamics.

\subsection{Estimates Based on Noncausal Autoregressions}

The starting point of our procedure of estimating the NKPC is an adequate, potentially noncausal AR model for demeaned inflation. Following the model selection procedure outlined in Section 2.2, we first specify a Gaussian autoregression with serially uncorrelated errors and check whether the residuals are normally distributed. As discussed above, it is the deviations from normality that facilitate identification of the parameters of interest. To that end we use the Ljung-Box autocorrelation and 
Jarque-Bera normality tests. For $\pi_{t}^{G D P}$, five lags are required, while for $\pi_{t}^{C P I}$, a fourth-order AR model is deemed sufficient. For all residual series, the Jarque-Bera test clearly rejects the null hypothesis of normally distributed errors, with p-values close to zero. Observed excess kurtosis suggests that a fat-tailed error distribution, such as Student's $t$ distribution with $\nu$ degrees of freedom might be suitable. This reconfirms the previous findings of Lanne and Saikkonen (2011a) and Lanne et al. (2011).

After specifying the adequate autoregressive orders, the next step is finding the correct orders of causal and noncausal lag polynomials, $r$ and $s$, respectively. To that end, we estimate all $\operatorname{AR}(r, s)$ models with $t$-distributed errors where the sum of $r$ and $s$ equals five for $\pi_{t}^{G D P}$ and four for $\pi_{t}^{C P I}$. The values of the maximized loglikelihood functions are presented in Table 2. For both series, a mixed model involving both leads and lags is selected. Hence, the purely forward-looking NKPC (3) gets little support, as lagged inflation always seems to carry at least some significance. The selected models are $\operatorname{AR}(2,3)$ and $\operatorname{AR}(3,1)$ for $\pi_{t}^{G D P}$ and $\pi_{t}^{C P I}$, respectively. The insignificance of additional leads and lags reported in Table 2 attests to the adequacy of the selected noncausal AR models. The quantile-quantile plots of the residuals depicted in Figure 2 indicate the good fit of Student's $t$ distribution; especially for inflation based on the GDP deflator the fit is excellent also at the tails. The estimated small values of the degree-of-freedom parameter $\nu$ in Table 3 also lend support to a leptokurtic error distribution.

Because a mixed noncausal model is selected for each inflation series, we proceed with the estimation of the hybrid NKPC (4). The estimation results are presented in Table 3. The estimates of $\gamma_{b}$ and $\gamma_{f}$ are significant at conventional significance levels in both cases. Furthermore, for both inflation series, the estimates clearly indicate dominance of forward-looking behavior: the estimates of $\gamma_{f}$ substantially exceed those of $\gamma_{b}$. All estimates also fall in the shaded area of Figure 1. The $\operatorname{AR}(2,3)$ process 
selected for the GDP deflator inflation has one unstable and two stable characteristic roots. Of the stable roots, one is negative and one is positive. The estimates in Table 3 correspond to the positive stable root. The estimates of $\gamma_{f}$ and $\gamma_{b}$ corresponding to the negative stable root equal 3.465 and -2.829 , respectively. Because the former exceeds unity and the latter is negative, they can be precluded on theoretical grounds, and we have, in practice, unique identification. As far as the CPI inflation is concerned, there is only one stable and one unstable real characteristic root, which quarantees identification.

The influence of lagged inflation is indeed minor despite the fact that $\gamma_{b}$ is statistically significant. This can be seen by computing the roots of the $\operatorname{AR}(r, s)$ process of inflation from equation (6) underlying the NKPC. For the GDP deflator inflation, the stable root equals 0.421 implying a "half-life" of a percentage rise in inflation of less than a quarter. For the CPI inflation, the stable root equals only 0.229 with an even shorter half-life. This is in line with the findings of Galí et al. (2005).

To gain futher insight, it is useful to relate the results to a structural model undelying the hybrid NKPC. Galí and Gertler (1999) assume that each firm is able to adjust ist price with a fixed probability $1-\delta$, and a fraction $1-\chi$ of the firms set their prices optimally, while the rest use a simple rule of thumb based on the recent history of aggregate price behavior. Galí and Gertler (1999) derive the mapping from the reduced-form parameters $\gamma_{f}, \gamma_{b}$ and $\lambda$ to the above-mentioned 'deep' parameters $\delta$, $\chi$ and the discount factor $\beta$. Because we have no estimate of $\lambda$, the deep parameters cannot be uniquely solved, but instead we consider the range of their values given plausible values of $\lambda$. According to the survey of Schorfheide (2008), estimates of $\lambda$ obtained in the previous literature are typically rather small, with the vast majority of them below 0.05. Therefore, we compute the ranges of the deep parameters corresponding to the values between 0.001 and 0.05 of $\lambda$. Here we discuss the estimates for the GDP deflator inflation; the corresponding results for the CPI inflation are 
similar. Irrespective of $\lambda$, the implied value of $\beta$ hovers around 0.95 , whereas both $\delta$ and $\chi$ decline monotonically as $\lambda$ increases. The probability of not being able to adjust prices, $\delta$, declines at a faster rate with a range from 0.890 to 0.728 . The estimated fraction of backward-looking firms, $\chi$, correspondingly, ranges from 0.367 to 0.314. Thus, the results seem to be quite robust with respect to $\lambda$ and well in line with the findings in the previous literature also in terms of the main structural theory underlying the hybrid NKPC.

All in all, our results thus lend strong support to the importance of forwardlooking behavior in determining inflation, in line with Galí et al. (2005). At the same time they suggest that lagged inflation also has a role to play. Compared to previous research, though, our approach is more general in that no driver of inflation needs to be prespecified. When identification is purely statistical, making use of deviations from normality of the error term, the results are not influenced by an arbitrarily measured marginal cost variable. We also completely avoid the problems caused by weak and noncausal instruments in GMM estimation. However, our results deviate from those obtained by methods robust to weak instruments; as mentioned in the Introduction, Nason and Smith (2008a), inter alia, have found little evidence of forward-looking behavior with these methods. A potential explanation of the differences is that some of the instruments used in the previous literature are not only weak but also noncausal, which is not remedied by the robust methods.

\subsection{What Drives Inflation?}

As discussed in the Introduction, finding the correct variable driving the process of inflation is crucial for identification in conventional GMM and ML estimation approaches put forth in the previous literature. As our approach only makes use of the inflation series, it facilitates independently extracting the most plausible driver 
of inflation assuming the validity of the best-fitting NKPC. In other words, once the NKPC has been estimated, $\lambda x_{t}$ can be solved as

$$
\lambda x_{t}=\pi_{t}-\widehat{\gamma}_{f} E_{t} \pi_{t+1}-\widehat{\gamma}_{b} \pi_{t-1}
$$

where $\widehat{\gamma}_{f}$ and $\widehat{\gamma}_{b}$ are the ML estimates, and $E_{t} \pi_{t+1}$ can be computed as a forecast from the estimated $\operatorname{AR}(r, s)$ as shown by Lanne et al. (2010). Neither the marginal cost variable $x_{t}$ nor the coefficient $\lambda$ as such are not, of course, identifiable, but the time series of $\lambda x_{t}$ are informative about the properties of the implied drivers of the inflation series.

The driving processes of the two inflation series (scaled by their respective $\lambda \mathrm{s}$ ) implied by our estimates are depicted in Figure 3. They exhibit relatively low persistence, and hence, clearly deviate from the labor's share and output gap series, the principal candidate $x$-series considered in the previous literature. This finding is consistent with our results as well as those of Lanne and Saikkonen (2011a) and Lanne et al. (2011) that inflation persistence mostly results from agents' forward-looking behavior. Persistence is thus mostly intrinsic instead of being inherited from a persistent driving process. Also the recent results of Fuhrer (2006) and Sbordone (2007) suggest a minor role for the driving process as a source of inflation persistence albeit they use very diferent methods.

\section{Conclusion}

We have proposed a new estimation method of the NKPC that avoids a number of problems of the GMM commonly employed in the single-equation framework. In particular, no marginal cost proxy is required, and the detrimental effects of potentially weak or noncausal instruments are eliminated. Our estimator is based on specifying a potentially noncausal univariate autoregressive model for inflation whose identification relies on non-Gaussian errors. If no noncausality is detected, inflation dynamics 
are necessarily backward-looking, and the NKPC is refuted. On the other hand, finding noncausality, facilitates estimation of the NKPC and assessment of the relative importance of backward-looking and forward-looking behavior in determining inflation.

We applied the proposed procedure to two quarterly U.S. inflation series. In each case, the results lend support to both forward-looking and backward-looking dynamics, with the former clearly dominating. As we have prespecified no marginal cost proxy driving the inflation, the model facilitates computing the most plausible driving process given the estimated parameter values. The properties of these processes indicate that inflation persistence is likely to be intrinsic as opposed to being inherited from a persistent driving process.

\section{References}

Andrews, B., R.A. Davis, and F.J. Breidt (2006). Maximum likelihood estimation for all-pass time series models. Journal of Multivariate Analysis 97, 1638-1659.

Cecchetti, S.G. and G. Debelle (2006). Has the inflation process changed? Economic Policy, April 2006, 311-352.

Calvo, G. (1983). Staggered prices in a utility-maximizing framework. Journal of Monetary Economics 12, 383-398.

Fuhrer, J.C. (2006). Intrinsic and inherited inflation persistence. International Journal of Central Banking 2, 49-86.

Galí, J., and M. Gertler (1999). Inflation dynamics: A structural econometric approach. Journal of Monetary Economics 44, 195-222. 
Galí, J., M. Gertler, and J.D. López-Salido (2005). Robustness of the estimates of the hybrid New Keynesian Phillips curve. Journal of Monetary Economics $52,1107-1118$.

Lanne, M., and P. Saikkonen (2011a). Noncausal autoregressions for economic time series. Journal of Time Series Econometrics, forthcoming.

Lanne, M., and P. Saikkonen (2011b). GMM estimation with noncausal instruments. Oxford Bulletin of Economics and Statistics, forthcoming.

Lanne, M., A. Luoma, and J. Luoto (2011). Bayesian model selection and forecasting in noncausal autoregressive models. Journal of Applied Econometrics, forthcoming.

Lanne, M., J. Luoto, and P. Saikkonen (2010). Optimal forecasting of noncausal autoregerssive time series. HECER Discussion Paper No. 286.

Nason, J.M., and G.W. Smith (2008a). Identifying the new Keynesian Phillips curve. Journal of Applied Econometrics 23, 525-551.

Nason, J.M., and G.W. Smith (2008b). The new Keynesian Phillips curve: Lessons from single-equation econometric estimation. Economic Quarterly 94, 361-395.

Newey, W., and K. West (1994). Automatic lag selection in covariance matrix estimation. Review of Economic Studies 61, 631-653.

Rudd, J., and K. Whelan (2005a). New tests of the new-Keynesian Phillips curve. Journal of Monetary Economics 52, 1167-1181.

Rudd, J., and K. Whelan (2005b). Does labor's share drive inflation? Journal of Money, Credit, and Banking 37, 297-312. 
Rudd, J., and K. Whelan (2007). Modeling inflation dynamics: A critical review of recent research. Journal of Money, Credit, and Banking, Supplement to Vol. $39,155-170$.

Sbordone, A.M. (2007). Inflation persistence: Alternative interpretations and policy implications. Journal of Monetary Economics 54, 1311-1339.

Schorfheide, F. (2008). DSGE model-based estimation of the new Keynesian Phillips curve. Economic Quarterly 94, 397-433.

Stock, J.H., and M.W. Watson (1999). Forecasting inflation. Journal of Monetary Economics 44, 293-335.

Stock, J.H., and M.W. Watson (2009). Phillips Curve Inflation Forecasts. Ch. 3 in Understanding Inflation and the Implications for Monetary Policy (2009), J. Fuhrer, Y. Kodrzycki, J. Little, and G. Olivei (eds). Cambridge: MIT Press: $101-186$. 


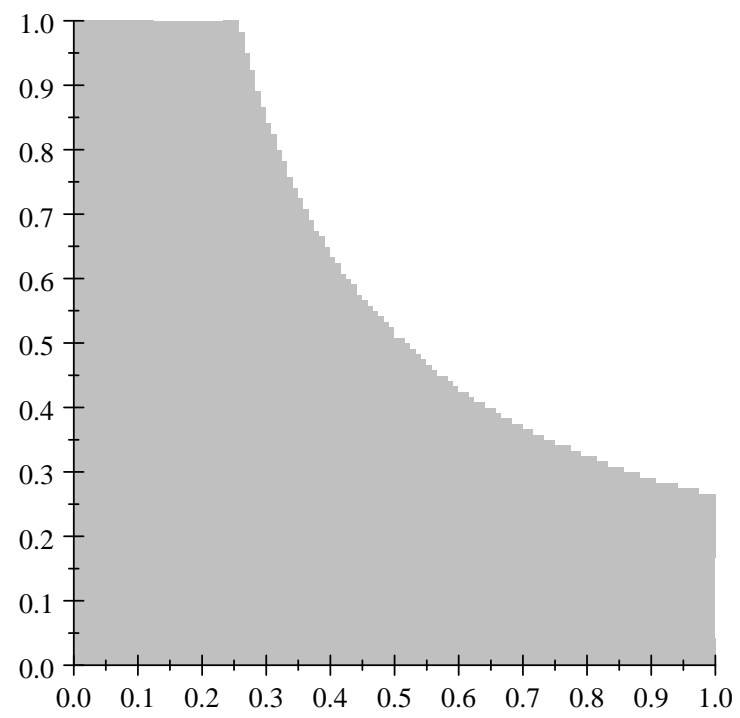

Figure 1: The values of $\gamma_{f}$ (x-axis) and $\gamma_{b}$ (y-axis) that produce real roots $\phi$ and $\varphi^{*}$ in (6). 

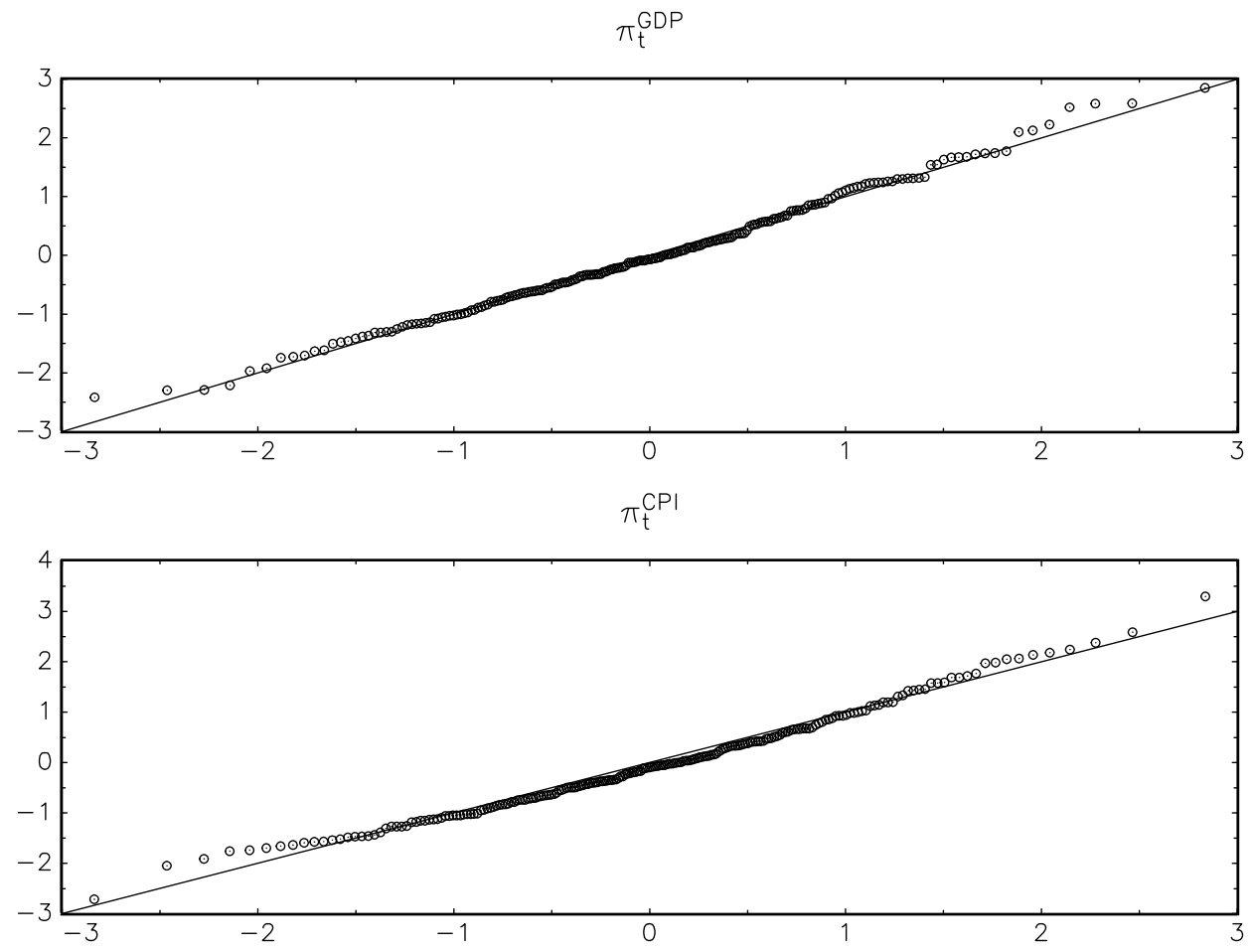

Figure 2: Quantile-quantile plots of the residuals of the noncausal AR models for the U.S. inflation series. 

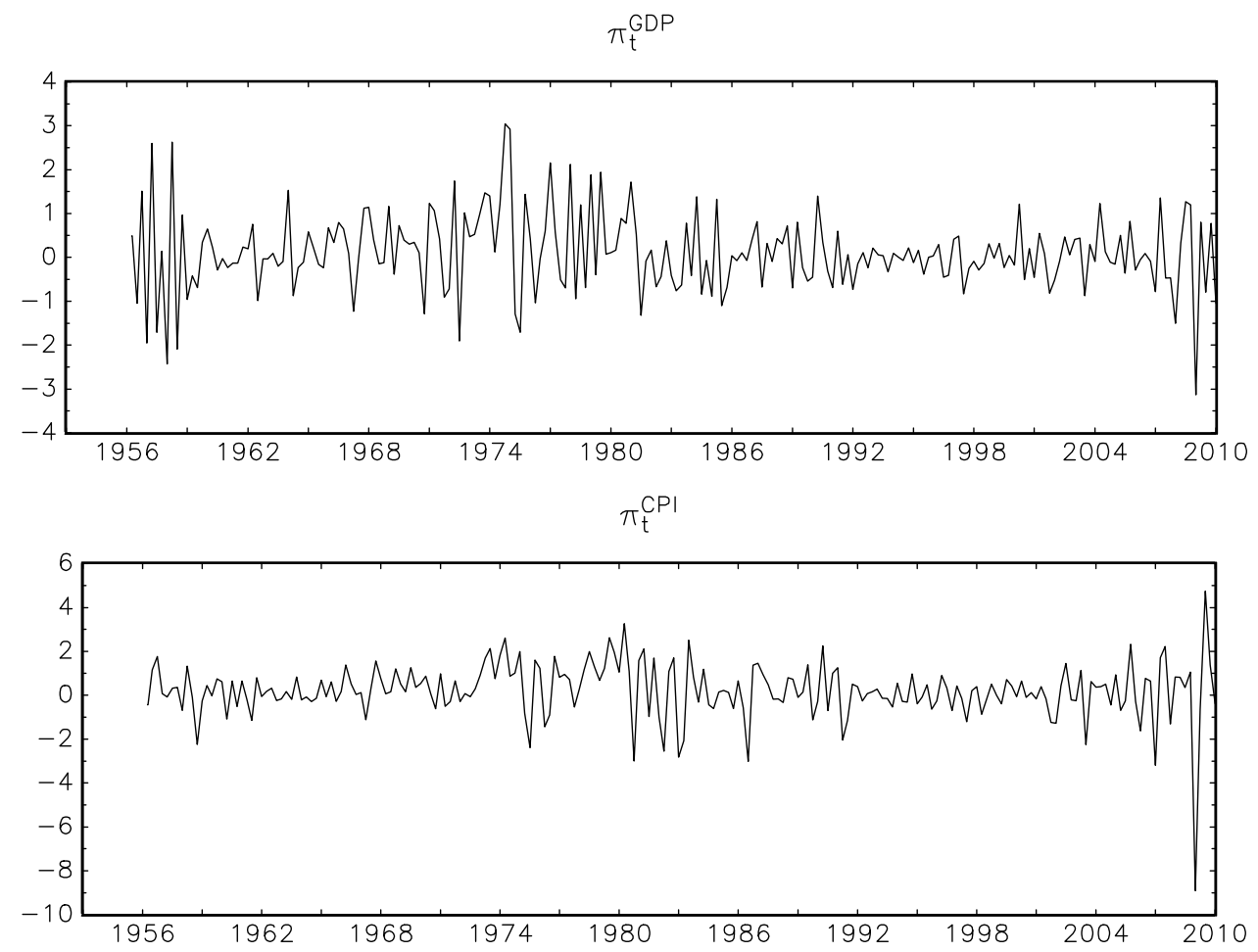

Figure 3: The drivers of the inflation series implied by the estimated new Keynesian Phillips curves (scaled by $\lambda$ ). 
Table 1: GMM estimates of the U.S. NKPC (4).

\begin{tabular}{|c|c|c|c|c|c|c|c|}
\hline & \multirow[b]{3}{*}{ Instruments } & \multicolumn{6}{|c|}{$x_{t}$} \\
\hline & & \multicolumn{3}{|c|}{ Real unit labor cost } & \multicolumn{3}{|c|}{ Detrended output } \\
\hline & & $\gamma_{b}$ & $\gamma_{f}$ & $\lambda$ & $\gamma_{b}$ & $\gamma_{f}$ & $\lambda$ \\
\hline \multirow[t]{8}{*}{$\pi_{t}^{G D P}$} & $z_{1 t}$ & -0.070 & 1.088 & 3.679 & -2.055 & 3.590 & -26.602 \\
\hline & & $(0.365)$ & $(0.410)$ & $(1.883)$ & $(6.554)$ & $(8.166)$ & $(70.291)$ \\
\hline & $z_{2 t}$ & 0.259 & 0.729 & 4.027 & 0.031 & 0.989 & -3.744 \\
\hline & & $(0.095)$ & $(0.098)$ & $(1.261)$ & $(0.228)$ & $(0.259)$ & $(2.247)$ \\
\hline & $z_{3 t}$ & -0.092 & 1.114 & 3.576 & -0.005 & 1.026 & -3.986 \\
\hline & & $(0.273)$ & $(0.302)$ & $(1.549)$ & $(0.336)$ & $(0.409)$ & $(4.151)$ \\
\hline & $z_{4 t}$ & -0.151 & 1.174 & 3.779 & -1.224 & 2.549 & -17.615 \\
\hline & & $(0.272)$ & $(0.303)$ & $(1.779)$ & $(2.485)$ & $(3.094)$ & (26.117) \\
\hline \multirow[t]{8}{*}{$\pi_{t}^{C P I}$} & $z_{1 t}$ & -0.015 & 1.106 & 5.089 & -0.141 & 1.356 & -8.059 \\
\hline & & $(0.241)$ & $(0.346)$ & $(3.480)$ & $(0.626)$ & $(0.923)$ & (11.167) \\
\hline & $z_{2 t}$ & 0.146 & 0.903 & 2.922 & 0.254 & 0.775 & -1.333 \\
\hline & & $(0.087)$ & $(0.123)$ & $(2.567)$ & $(0.105)$ & $(0.148)$ & $(3.014)$ \\
\hline & $z_{3 t}$ & -0.018 & 1.093 & 5.386 & 0.725 & 0.056 & 7.617 \\
\hline & & $(0.239)$ & $(0.343)$ & $(2.765)$ & $(0.189)$ & $(0.245)$ & $(3.728)$ \\
\hline & $z_{4 t}$ & -0.020 & 1.109 & 5.396 & 0.075 & 1.020 & -4.299 \\
\hline & & $(0.167)$ & $(0.225)$ & $(3.449)$ & $(0.248)$ & $(0.364)$ & $(0.481)$ \\
\hline
\end{tabular}

Sample period: 1955:1-2010:3. The figures in parentheses are Newey-West standard errors with automatic lag selection (Newey and West (1994)). Instrument set $z_{1 t}$ consists of $\pi_{t-1}, x_{t-1}, x_{t-2}$ and $x_{t-3}$. Sets $z_{2 t}, z_{3 t}$, and $z_{4 t}$ contain, in addition, $w i_{t-1}$ and $w i_{t-2}, c p_{t-1}$ and $c p_{t-2}$, and $t s_{t-1}$ and $t s_{t-2}$, respectively. A constant is included in all models. 
Table 2: Estimation results of the $\operatorname{AR}(r, s)$ models for the inflation series.

\begin{tabular}{|c|c|c|c|c|c|}
\hline \multicolumn{3}{|c|}{$\pi_{t}^{G D P}$} & \multicolumn{3}{|r|}{$\pi_{t}^{C P I}$} \\
\hline$r$ & $s$ & Log likelihood & $r$ & $s$ & Log likelihood \\
\hline 0 & 5 & -325.171 & 0 & 4 & -409.262 \\
\hline 1 & 4 & -320.588 & 1 & 3 & -404.941 \\
\hline 2 & 3 & -319.922 & 2 & 2 & -404.199 \\
\hline 3 & 2 & -324.744 & 3 & 1 & -403.611 \\
\hline 4 & 1 & -322.727 & 4 & 0 & -405.976 \\
\hline 5 & 0 & -326.809 & & & \\
\hline & $\left(r^{*}+1, s\right)$ & 0.209 & & & 0.725 \\
\hline & $\left(r, s^{*}+1\right)$ & 0.942 & & & 0.118 \\
\hline
\end{tabular}

The values of the maximized log-likelihood function of $\mathrm{AR}(r$, $s$ ) models for the different inflation series. The rows labeled $\mathrm{AR}\left(r^{*}+1, s\right)$ and $\operatorname{AR}\left(r, s^{*}+1\right)$ report the p-values of the Wald significance test of the coefficient of an additional lag and lead in the selected model, respectively. 
Table 3: Estimation results of the new Keynesian Phillips curves based on the U.S. inflation series.

\begin{tabular}{lll}
\hline & $\pi_{t}^{G D P}$ & \multicolumn{1}{c}{$\pi_{t}^{C P I}$} \\
\cline { 2 - 3 } AR Model & $\operatorname{AR}(2,3)$ & $\mathrm{AR}(3,1)$ \\
$\gamma_{b}$ & 0.302 & 0.189 \\
& $(0.099)$ & $(0.060)$ \\
$\gamma_{f}$ & 0.675 & 0.768 \\
& $(0.086)$ & $(0.057)$ \\
$\sigma$ & 1.154 & 1.917 \\
& $(0.108)$ & $(0.356)$ \\
$\nu$ & 4.527 & 3.010 \\
& $(1.490)$ & $(0.706)$
\end{tabular}

The row labeled AR Model gives the bestfitting $\operatorname{AR}(r, s)$ model that the estimation of the NKPC is based on. $\sigma$ and $\nu$ are the scale and degree-of-freedom parameters of the error distribution, respectively. The figures in parentheses are ML standard errors based on the Hessian matrix. 